International Journal on Design and Manufacturing Technologies, Vol.3, No.1, January 2009

\title{
TOLERANCES OF LINEAR, ANGULAR SIZED PARTS AND FEEDERS FOR RELIABLE ASSEMBLY PROCESSES IN PRECISION ENGINEERING
}

\author{
Ryspek Usubamatov ${ }^{1}$, Thoguluva Raghavan Vijayaram², Hamid F. ${ }^{3}$, Usubamatova A. \\ ${ }^{1,3,4}$ School of Manufacturing Engineering, University Malaysia Perlis, Malaysia \\ ${ }^{2}$ Department of Manufacturing Processes, Universiti Teknikal Malaysia Melaka, Malaysia \\ Email: 1 ryspek@unimap.edu.my
}

\begin{abstract}
The failure of functioning feeders and assembling mechanisms can result in major productivity losses of machines. Predicting of reliability of an automatic machines work has become an important part of precision engineering and involves mathematical approaches and calculation. The reliability of work of automated high-speed assembly mechanisms depends from the accuracy and tolerances of linear and angular sizes of parts and their dispositions relatively to elements of devices. This paper presents mathematical modeling for calculation of tolerances of assembled parts and feeder elements geometry, which results on reliability of the assembling and feeding processes in automatic machines. It also discusses about the computing of the optimal geometrical parameters of the feeding and assembling units that allows for mechanism to work in proper mode. Various mathematical dependencies derived for calculation of the limited size of the feeder's components, tolerances of parts and tolerances of linear and angular orientation for assembled parts that allow avoid the part sticking into feeders or brakes of assembling processes. Besides, a case study has undertaken to calculate the value of tolerances of feeder elements geometry and assembled parts for reliable assembly processes.
\end{abstract}

Key words: Assembling Process, Feeders, Reliability, Tolerances

\section{INTRODUCTION}

Operating experience of high productive automatic machines and automatic transfer lines for processing and assembly of cylindrical, flat and ring parts like pins, washers, bushes, shaft, etc. shows that their reliability in many cases need to be enhanced [1]. Most of failures or brakes of such as machines like transport mechanisms, collectors, stores, etc, that constructed in the form of chutes, trays, axial guide pins, axes, and platens [2]. The assembly mechanisms and units have also problems of reliability that substantially influences on productivity of the technological equipment [3]. Transporter, collectors and assembly systems can include several of chutes, and axial guide units on which cylindrical, flat, ring type details moved by rolling or sliding due to gravity forces and forces of machines. Parts can move in many variants by single piece or parties, periodically or continuous streams depending on demands of manufacturing conditions [4]. Brakes of functioning of such devices, in the form of jamming or sticking of assembled parts into mechanisms, lead to stoppages of the manufacturing processes [5].

In spite of constructive simplicity of feeders, collectors and assembly units, the practice of their application shows that brakes in the form of jamming parts in guide mechanisms occur by many reasons. The main reasons are changing of the sizes of chute boards, axial guides and moved parts, pollution, magnetization and not coordinated moving of parts, etc. In transport mechanisms such as simple chutes for rolling or sliding parts and assembly mechanisms for joining of type a shaft - bush, the jamming of parts occurs basically as a result of a deviation of axes of assembled parts during the initial moment of transporting, and joining of assembly elements take place [6].

Jamming of parts in feeders and assembly mechanisms has probabilistic character of sticking that influences the deviation of the geometrical sizes of the guide mechanisms, transported and assembled details, their uncontrollable and non-oriented movement, etc.

Calculations of reliability work of such mechanisms should be based on definition of limited geometrical parameters of elements of feeders and assembly units providing their qualitative work. The known approaches and mathematical presentations of boundary conditions of reliable motion of parts in chutes, guide ways, and guide rods or pegs have quite artificially complex dependencies and Equations in some cases are incorrect [7].

This article presents new mathematical approaches for the guaranteed movement by rolling or sliding the cylindrical, flat and ring parts in chutes and on axial guides as function of the limited sizes guides and parts [8]. Mathematical dependencies of limited sizes that guaranteed work of such devices are defined under the following scheme.

Let's consider the conditions of jamming for cylindrical, flat and ring type details with rectangular and round cross section at their movement by rolling or sliding in chutes and on inclined axial guide when its angle of an inclination in the form of the platen is more than angle of a sliding friction between surfaces of a part and a guide. For the rolling and sliding cylindrical and flat parts jamming 
occurs in one plane. Jamming of suspended parts can occur in two planes [9]. In a plane of action of weight forces passing through the contact point of the suspended ring on inclined axial guide and in a plane passing through an axis of inclined guide and perpendicular to a plane of weight forces of rings as a result of a turn of the suspended ring on axial guide. The second case of jamming of rings can be generalized in a case of a vertical arrangement axial guide when rings fall under action of weight forces [10].

\section{CALCULATION OF BOUNDARY CONDITIONS OF FEEDING OF CYLINDRICAL AND FLAT PARTS}

Fig.1 shows typical design of parts in manufacturing area that transported in chutes [5].

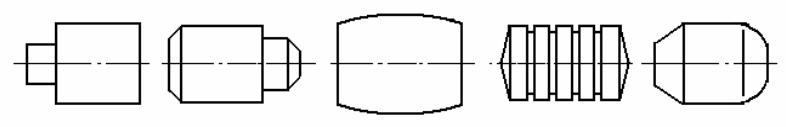

Fig. 1. Typical design of parts in manufacturing area that transported in chutes

The sizes that guaranteed the reliable transportation of parts into chute can be found on a base of known parameters of chutes and parts (Fig. 2). Let's present the simple part twisted into the chute and contacted with its board [7]. The angle è expresses parameters of the chute and the twisted simple part in the chute (Fig. 2, I) by next:

$\operatorname{tg} \theta=\mathrm{C} / \mathrm{W}, \mathrm{C} 2=\mathrm{L} 2-\mathrm{W} 2, \mathrm{~L} 2=\mathrm{H} 2+\mathrm{D} 2$

where $\mathrm{C}$ is distance between the point of contact the right end of part's diagonal $L$ with the chute board and the point of intersection the perpendicular from the point of contact the left end of part's diagonal with chute's board; $W$ is the width of the chute's canal; $H$ is height of the part; $D$ is width of the part; $\operatorname{tg}$ è $=f$ that presents factor friction between part's and chute's boards surfaces; $\ddot{A}$ is clearance between the chute' boards and the part.

After substitutions and transformations the Equation of the limited of size of the chute width when the twisted part can stick is expressed by next (Fig. 2, I)

$$
W=\sqrt{\frac{H^{2}+D^{2}}{1+f^{2}}}
$$

Equation (1) can be used for calculation of limited width of the chute's canal, which less sizes can guarantee the motion cylindrical parts by rolling and flat parts by sliding in the chute without jamming them in feeders of transport system. It is easy to imagine the
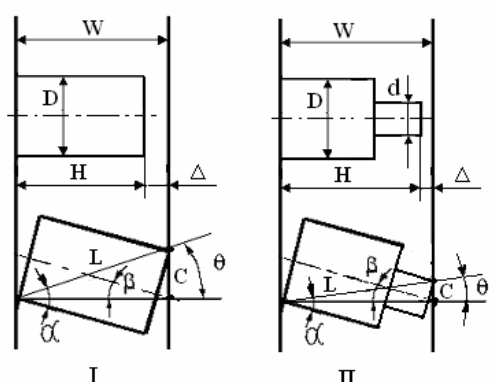

II

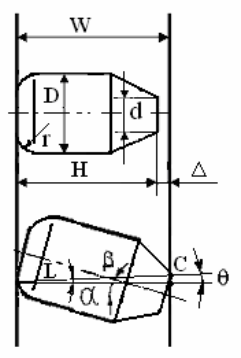

III
Fig. 2. The jam of parts in the chute.

sticking process of geometrically more complex part design in the chute. In addition, it is not a problem to determine necessary calculated parameters for parts; particularly the length of part's diagonal L (Fig. 2, II, III) and develop Equation of limited width size of the chute on a base elementary geometry and mathematics.

\section{CALCULATION OF BOUNDARY CONDITIONS OF FEEDING RING TYPE PARTS AND ASSEMBLY OF A BUSH AND SHAFT TYPE PARTS}

Fig. 3 shows typical design of ring type parts in manufacturing area that transported on axial guides [6].
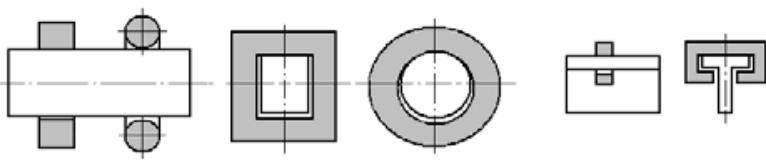

Fig. 3. Typical design of ring type parts in manufacturing area that transported on axial guides

The further mathematical calculations show, that Equations of calculation of boundary conditions for ring jamming on axial guide have one expression for all cases of movement of rings into mechanisms with axial guides. Let's consider the scheme of calculation for ring jamming conditions on axial guides (Fig. 4).

For rings with round cross-section jamming occurs under the same conditions mentioned above. We shall present the angle è through geometrical parameters of a ring with round cross-section and a guide in a following kind (Fig. 4, I).

tgè $=, \frac{A}{D}$ whereA expressed from triangle abc (Fig. 4, I), where $A=\sqrt{L^{2}-D^{2}}$, but $L$ we should find from the triangle agk $(\mathrm{L} / 2) 2=(\mathrm{gk}) 2+(\mathrm{D} / 2) 2$ The segment $\mathrm{gk}$ is defined from the triangle gek, $(\mathrm{gk}) 2=[(\mathrm{C} / 2)+(\mathrm{d} / 2)] 2-[(\mathrm{D} / 2)+(\mathrm{d} / 2)] 2$.

After substitution and transformation we shall produce the expression of the segment $L$

$$
\left(\frac{L}{2}\right)^{2}=\left(\frac{C}{2}+\frac{d}{2}\right)^{2}-\left(\frac{D}{2}+\frac{d}{2}\right)^{2}+\left(\frac{D}{2}\right)^{2}
$$




$$
L^{2}=(C+d)^{2}-(D+d)^{2}+D^{2},
$$

then after substitution and simplification we can get the expression of the segment $A$

$$
\begin{aligned}
& A=\sqrt{(C+d)^{2}-(D+d)^{2}+D^{2}-D^{2}}= \\
& \sqrt{(C+d)^{2}-(D+d)^{2}} \\
& \operatorname{tg} \theta=\frac{\sqrt{(C+d)^{2}-(D+d)^{2}}}{D} \text { or } \\
& \mathrm{f}=\frac{\sqrt{(C+d)^{2}-(D+d)^{2}}}{D}
\end{aligned}
$$

, then

Accepting tgè $=\mathrm{f}$, doing substitutions and transformations, we shall express the maximum diameter $D$ of the platen, at which occurs jamming of a ring with round-cross section on the guide. Actual diameter of the platen should be less then its maximum diameter that enables avoiding the jamming of a ring on the platen.

$$
\begin{aligned}
& f=\frac{\sqrt{(C+d)^{2}-(D+d)^{2}}}{D} \\
& D f=\sqrt{(C+d)^{2}-(D+d)^{2}} \\
& (D f)^{2}=(C+d)^{2}-(D+d)^{2} \\
& D^{2} f^{2}+(D+d)^{2}-(C+d)^{2}=0 \\
& \left(1+f^{2}\right) D^{2}+2 D d+d^{2}-(C+d)^{2}=0 \\
& D=\frac{-2 d \pm \sqrt{4 d^{2}-4\left(1+f^{2}\right)\left[d^{2}-(C+d)^{2}\right]}}{2\left(1+f^{2}\right)}= \\
& \frac{-d \pm \sqrt{d^{2}-\left(1+f^{2}\right)\left[d^{2}-(C+d)^{2}\right]}}{\left(1+f^{2}\right)} \\
& \text { or } D \leq \frac{-d \pm \sqrt{d^{2}-\left(1+f^{2}\right)\left[d^{2}-(C+d)^{2}\right]}}{\left(1+f^{2}\right)}
\end{aligned}
$$

where $d$ is the diameter of a ring cross-section, other parameters are similar to parameters of a ring with rectangular cross-section and the platen considered above.

For rings with rectangular cross-section jamming occurs in case of contact of a ring by two diametrically opposite points of surfaces on the guide platen (Fig.4.II)

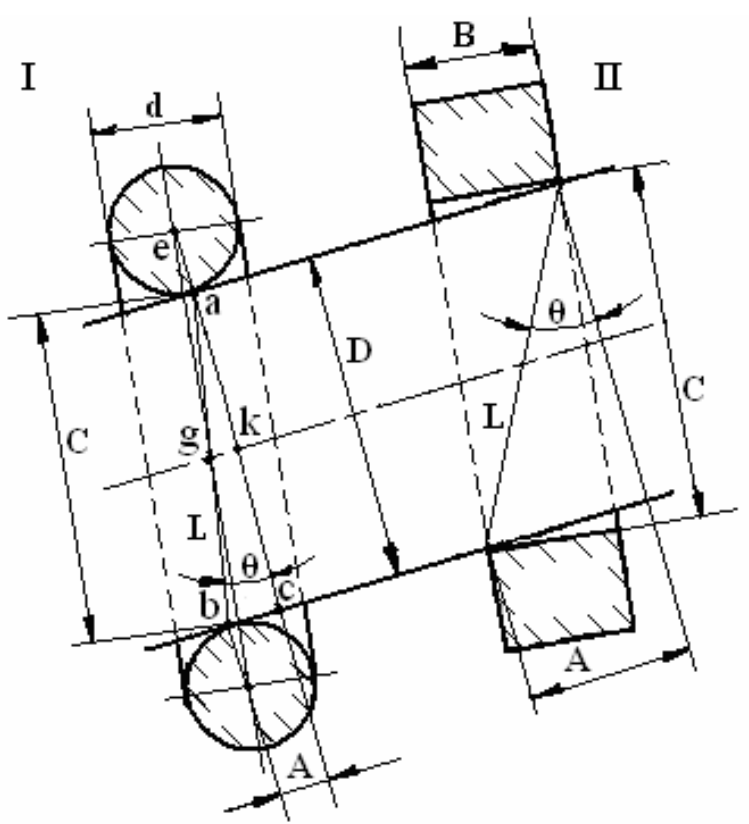

Fig. 4. The jam of parts on the axial guide

Thus the angle è of the jamming formed by line $L$ connected the contact points of a ring with the platen and a line of a perpendicular to an axis of the platen, should be equal or less an angle of a sliding friction of a detail on surface guide.

We shall present the angle è through geometrical parameters of a ring and guide in a following kind. $\operatorname{tg} \theta=$ $\mathrm{A} / \mathrm{D}$, where $\mathrm{A}=\sqrt{L^{2}-D^{2}}$, but $\mathrm{L} 2=\mathrm{B} 2+\mathrm{C} 2$, then $\mathrm{A}$ $=\sqrt{B^{2}+C^{2}-D^{2}} \quad, \quad$ and finally $\operatorname{tg} \theta=\frac{\sqrt{B^{2}+C^{2}-D^{2}}}{D}$, where $B$ is the width of a ring

$C$ is the internal diameter of a ring, $D$ is the diameter of the guide platen $(C>D)$.

Accepting $\operatorname{tg} \theta=f(f$ is factor of a sliding friction) we shall present expression of the maximal diameter of platen $D$ at which occurs jamming a ring with rectangular section on the guide.

After substitution $\mathrm{f}=\sqrt{B^{2}+C^{2}-D^{2}}$, then

$\mathrm{D} \leq \sqrt{\frac{B^{2}+C^{2}}{1+f^{2}}}$ 
The scheme of ring's jamming considered above on an axial guide of feeder for a ring type details can be used for a case of assembly process into mechanisms having joining of type a shaft and a bush. The given scheme of calculation of boundary conditions for assembly of elements can explain the reasons of jamming of platens in holes into joining with a clearance during the initial moment of assembly process and give maximum deviations of geometrical parameters of assembled elements by criterion of the guaranteed reliable assembly process of mechanical units.

Let's consider assembly process of joining with a clearance between a shaft and the bush [11, 12]. It is known that jamming of a shaft in a hole of the bush occurs at input to an angular deviation of an axis of a shaft concerning an axis of a bush's hole [13]. The scheme of jamming of a shaft in a bush's hole during assembly is presented in the Fig. 5.

The angle á of an inclination of a shaft's axis at initial depth $\mathrm{h}$ of input the shaft in the hole at which occurs its jamming in a bush's hole is defined under the following scheme. Diagonal $L$ connects a point of contact of a line of a shaft's end face and a cylindrical surface of hole of a bush with a diametrically opposite point of contact of a shaft's surface with a line of the end face of hole of a bush. The angle è is formed by the diagonal $L$ and the diameter line of hole of the bush. Further under the scheme of calculation (Fig.5) is defined

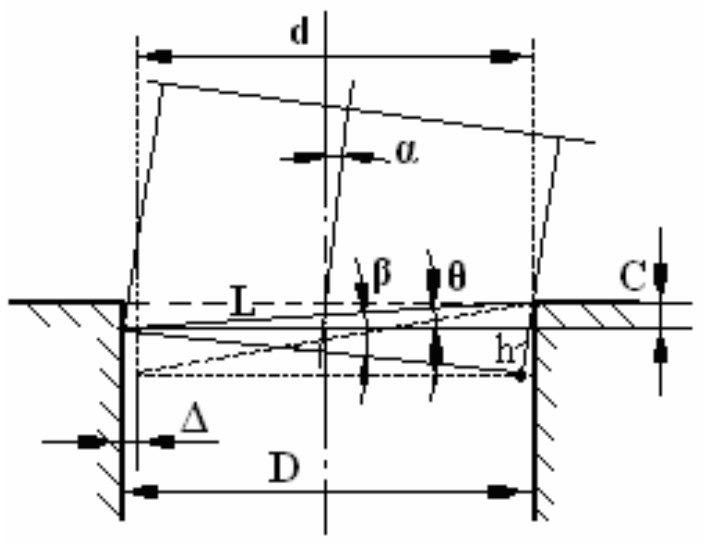

Fig. 5. The shaft sticks in the hole of bush.

$$
\begin{aligned}
& \operatorname{tg} \theta=\mathrm{f}=\mathrm{C} / \mathrm{D}, \text { Where } \quad C=\sqrt{L^{2}-D^{2}}, \\
& \text { then } \operatorname{tg} \theta=\sqrt{L^{2}-D^{2}} / \mathrm{D}, \\
& \text { where } L=\sqrt{h^{2}+d^{2}} \text {, and finally } \\
& \operatorname{tg} \theta=\mathrm{f}=\frac{\sqrt{h^{2}+d^{2}-D^{2}}}{D},
\end{aligned}
$$

$$
\begin{aligned}
& \text { after transformations we shall get } \\
& \begin{array}{l}
h=\sqrt{(d+\Delta)^{2}\left(1+f^{2}\right)-d^{2}} \\
\text {, but } \mathrm{D}=\mathrm{d}+\ddot{\mathrm{A}} \text {, then }
\end{array} \\
& h=\sqrt{D^{2}\left(1+f^{2}\right)-d^{2}}
\end{aligned}
$$

where $h$ is critical depth of input of a shaft into a bush's hole, at which jamming is occurred, if the depth of input of a shaft into a hole is more then critical, the jamming does not occur; $d$ is diameter of a shaft; $D$ is diameter of a bush's hole (Fig. 5); $\Delta$ is clearance between the shaft and the bush; other components presented above.

The limited angle a of a deviation of a shaft axis concerns an axis of a bush's hole at which a jamming takes place. This angle depends on four parameters namely from

a shaft diameter $d$; diameter of a bush's hole $D$ or clearance $\Delta$; depth $\mathrm{h}$ of input of a shaft into a bush's hole and from value $f$ of friction factor of a sliding.

The angle $a$ of a shaft inclination defined by next scheme (Fig.5). $\mathrm{a}=\beta-\theta, \cos \theta=\frac{d+\Delta}{L}$

where, $\cos \beta=d / L, L=\sqrt{h^{2}+d^{2}}$

After substitutions and transformation we shall get

$\alpha=\arccos \frac{d}{\sqrt{h^{2}+d^{2}}}-\arccos \frac{d+\Delta}{\sqrt{h^{2}+d^{2}}}$.

To get the dependency the change of angle $\alpha$ as function of the depth $h$ of input of a shaft into a bush's hole it is necessary substitute the expression $h$ (4) to Equation (5)

$$
\begin{aligned}
& \alpha=\arccos \frac{d}{\sqrt{(d+\Delta)^{2}(1+f)^{2}}}- \\
& \arccos \frac{d+\Delta}{\sqrt{(d+\Delta)^{2}(1+f)^{2}}}
\end{aligned}
$$

Jamming of a shaft at its input in a bush's hole occurs in case of when the angle $\theta$ of a shaft axis inclination becomes equal or less an angle of a sliding friction (friction factor of a sliding f). Hence the angle a of an shaft axis inclination should be less than the certain size (ap $<a$ ), allowing to make guaranty assembly of a shaft- bush type joining. At the big depth of input of a shaft into a bush's hole the angle of a shaft axis inclination will be insignificant, that proves to be true the Equations (4) and (5) and the probability of jamming is excluded.

All obtained Equations of limited size of chute's width or size of axial guides for ring type parts for jamming parts in some chutes, guide-ways, axial guides and so on presented for permanent sizes of feeders and transported 
parts. In real manufacturing engineering all sizes of all machines, units, parts and etc have some deviations that can be described by the law of normal distribution. In such case for reliable work of transport mechanisms it is necessary to calculate limited sizes of transport feeders and limited sizes of transported parts on a base of the law of normal distribution and probabilistic approach. Such approach is important for automatic machines in manufacturing area with high productive rate. Any jamming of parts in transport mechanisms and feeders lead to stop very expensive automatic machine and to decrease of efficiency use one. The probability calculation of jamming parts in transport mechanisms as function of probabilistic deviation of sizes for chutes and transported parts is also important but it is out of the scope consideration of this paper [7].

\section{CASE STUDY 1}

Compute the limited width of the chute for the part with height $\mathrm{H}=50 \mathrm{~mm}$, diameter $\mathrm{D}=20 \mathrm{~mm}$, and friction factor of a sliding $f=0.1$ at which jamming can take place. The limited width $W$ is defined by expression

$$
W=\sqrt{\frac{H^{2}+D^{2}}{1+f^{2}}}=\sqrt{\frac{50^{2}+20^{2}}{1+0.1^{2}}}=53.58 \mathrm{~mm}
$$

Making substitutions of initial data in the expression $\mathrm{W}$ and doing transformations, we shall receive value of limited size of the width of a chute $W=53.58 \mathrm{~mm}$, at which jamming part takes place at transport or assembly process. Fig. 6 shows the diagram of change the limited width $\mathrm{W}$ of a chute versus the change of diameter $\mathrm{D}$ of a part which leads to jamming part in the chute.

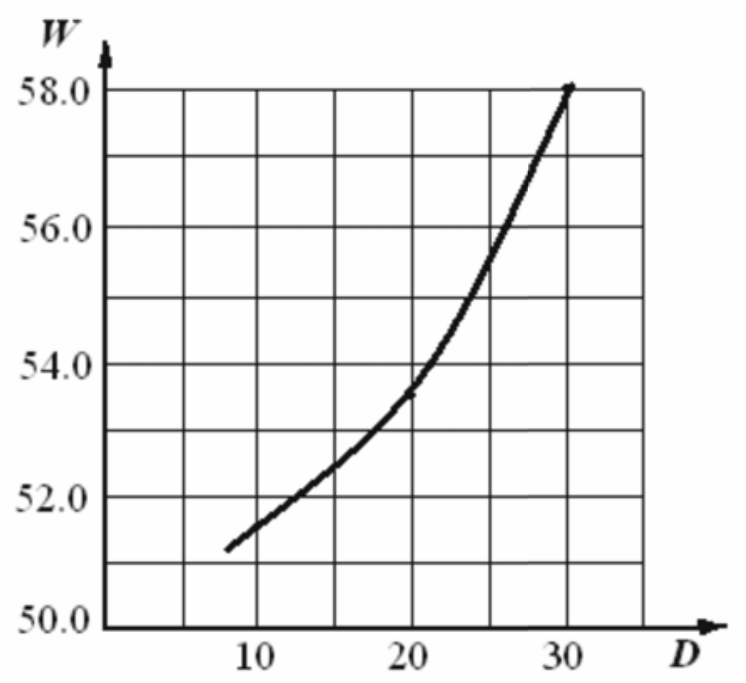

Fig. 6. The limited width $W$ of the chute versus change of the diameter $\mathrm{D}$ of the part, which leads to jamming part in the chute.
The diagram shows the less diameter of a part the less limited width of the chute, which the jamming of a part in the chute occurs.

\section{CASE STUDY 2}

(a) Compute the diameter of platen $D$ at which occurs jamming a ring with round cross-section on the guide. The ring parameters are next: diameter $d=20 \mathrm{~mm}$, inner diameter $\mathrm{C}=50 \mathrm{~mm}$, and friction factor of a sliding $\mathrm{f}=0.1$ at which jamming can take place. The limited diameter of platen $\mathrm{D}$ is defined by expression

$$
\begin{aligned}
& D \leq \frac{-d \pm \sqrt{d^{2}-\left(1+f^{2}\right)\left[d^{2}-(C+d)^{2}\right]}}{\left(1+f^{2}\right)}= \\
& \frac{-20 \pm \sqrt{20^{2}-\left(1+0.1^{2}\right)\left[20^{2}-(50+20)^{2}\right]}}{\left(1+0.1^{2}\right)}=
\end{aligned}
$$

\section{$49.64 \mathrm{~mm}$}

Making substitutions of initial data in the expression $D$ and doing transformations, we shall receive value of limited size of the diameter of platen $D=49.64 \mathrm{~mm}$, at which jamming ring takes place at transport or assembly process. Fig. 7 shows the diagram of change the limited diameter $\mathrm{D}$ of a platen versus the change of diameter $\mathrm{d}$ of a ring, which leads to its jamming on the platen.

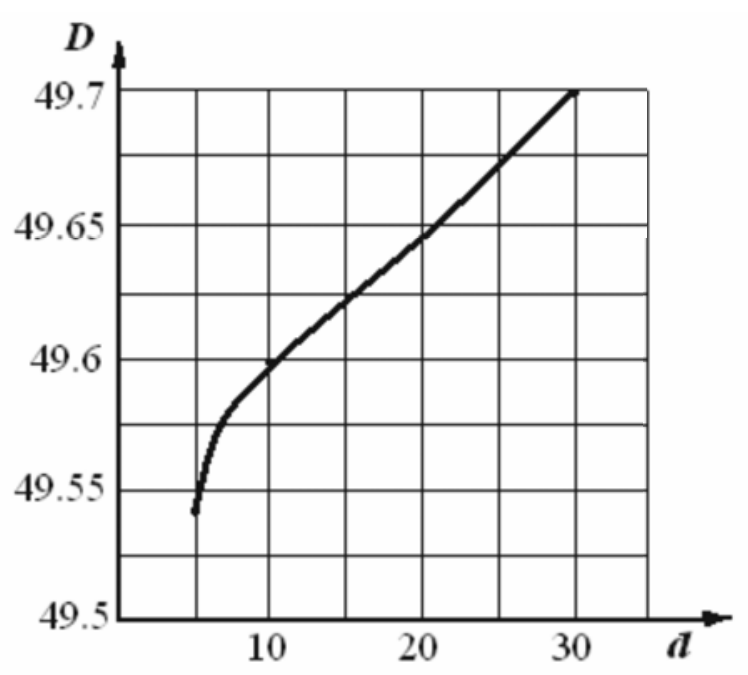

Fig. 7. The limited diameter $D$ of the platen versus of change of the diameter $d$ of the ring, which courses the ring stuck on the platen.

The diagram shows the less width of a ring the less limited diameter of the platen, which the jamming of a ring on the platen occurs.

(b) Compute the diameter of platen $D$ at which occurs jamming a ring with rectangular section on the guide. 
The ring parameters are next: width $\mathrm{d}=20 \mathrm{~mm}$, inner diameter $\mathrm{C}=50 \mathrm{~mm}$, and friction factor of a sliding $\mathrm{f}=0.1$ at which jamming can take place. The limited diameter of platen $\mathrm{D}$ is defined by expression

$$
D \leq \sqrt{\frac{B^{2}+C^{2}}{1+f^{2}}}=\sqrt{\frac{20^{2}+50^{2}}{1+0.1^{2}}}=53.58 \mathrm{~mm}
$$

Making substitutions of initial data in the expression $\mathrm{D}$ and doing transformations, we shall receive value of limited size of the diameter of platen $D=53.58 \mathrm{~mm}$, at which jamming ring takes place at transport or assembly process. Fig. 8 shows the diagram of change the limited diameter $\mathrm{D}$ of a platen versus the change of width $\mathrm{C}$ of a ring, which leads to its jamming on the platen.

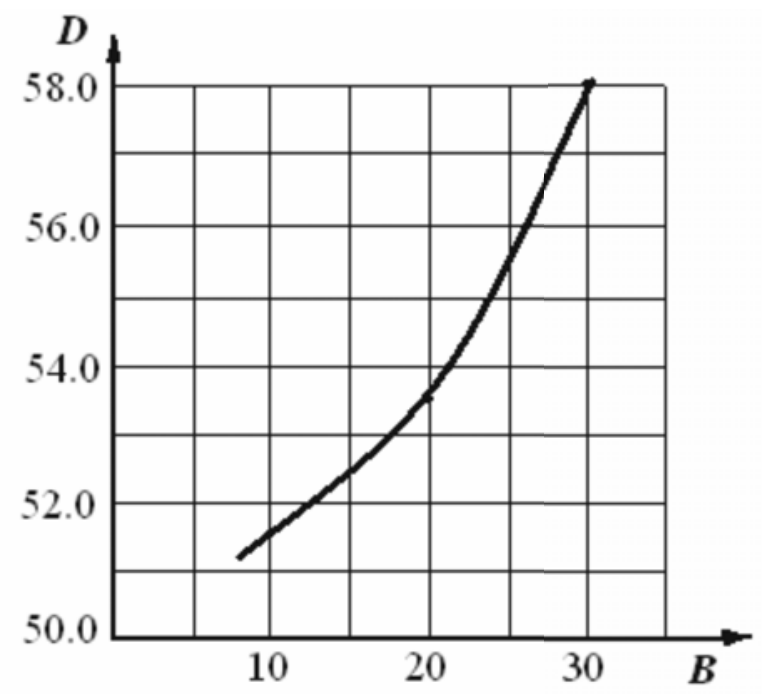

Fig. 8. The limited diameter $D$ of the platen versus change of the width $B$ of the ring, which courses the ring stuck on the platen

The diagram shows the less width of a ring the less limited diameter of the platen, which the jamming of a ring on the platen occurs.

\section{CASE STUDY 3}

For example, it is necessary to find the limited angle á of a deviation of a shaft axis with a diameter $d=20 \mathrm{~mm}$, at assembly with the bush that has the hole diameter

$D=20.01 \mathrm{~mm}$ or $\Delta=0.01 \mathrm{~mm}$, and friction factor of a sliding $f=0.1$ at which jamming can take place. The limited angle á is defined for limited size of input of a shaft into a bush's hole by expression

$$
\begin{aligned}
& \alpha=\arccos \frac{d}{\sqrt{(d+\Delta)^{2}(1+f)^{2}}}- \\
& \arccos \frac{d+\Delta}{\sqrt{(d+\Delta)^{2}(1+f)^{2}}}
\end{aligned}
$$

Making substitutions of initial data in the expression $\mathrm{h}$ and doing transformations, we shall receive value of limited size of input of a shaft into a bush's hole $h=2.098$ $\mathrm{mm}$. Substituting data of an example into the expression $\alpha$ and making calculations we shall receive $\alpha=0.2790=$ 16.7 ' the limited angle of a deviation of a shaft axis at which its jamming into a bush's hole takes place at assembly. Fig. 9 shows the diagram of change the limited angle $a$ of inclination of shaft versus the clearance $\Delta$ between shaft and hole of bush and dependency the critical depth $\mathrm{h}$ of input of a shaft into a hole of bush, which leads to jamming shaft in the hole.

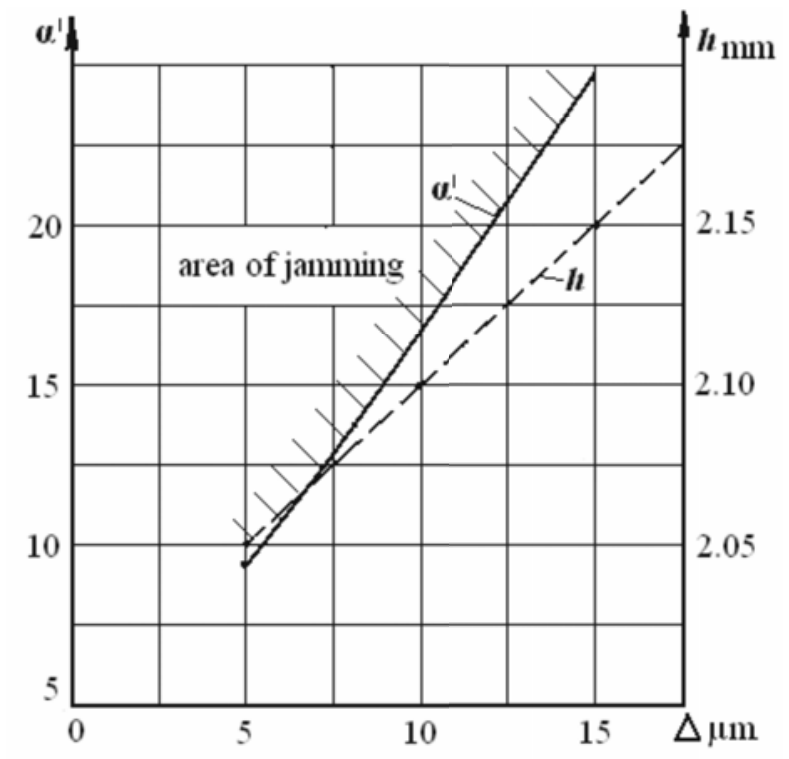

Fig. 9. The angle a of inclination of the shaft (minutes) and depth $h$ of input of a shaft into hole of a bush versus the clearance $\Delta$ (micrometers)

The diagrams show that the jamming of shaft in the hole occurs at initial depth of shaft in hole and need high coaxially of bush's and shaft's axis. Manual assembling needs to use simple devices to keep necessary coaxially to avoid the jamming. 


\section{RESULTS AND DISCUSSION}

Limited relative angle of a deviation of a shaft axis or a bush's axis calculated by the Equations $(1-3,5,6)$ allow establishing tolerances on a deviation of device axes of the assembly mechanisms, guarantee reliability and qualitative assembly of joining. It is obvious, that manual assembly does not guarantee exact axial orientation of joined details, hence their frequent jamming takes place. Practice of assembly operations confirm, that machine assembly better, in comparison with manual as gives a smaller axes deviation of a shaft and bush.

\section{CONCLUSIONS}

Equations (1) (2) and (3) for calculation of the limited sizes of the chute, guide platen, and also the Equations (5) and (6) for calculation of the maximal relative deviation of axes of a shaft and the bush give the limited sizes of parameters of feeders and assembly mechanisms, which are necessary for use at engineering calculations. Considering, that the factor of disorder of the detail sizes, the diameter of the guide platen and a deviation of axes of assembled parts under production conditions takes place, engineering calculations of reliability of feeding and assembly of parts can be based on the offered Equations for limited geometrical parameters of considered devices. Methods of calculation of limited geometrical parameters of feeding devices for parts with different geometry and for ring type parts on axial guide and assembly operations for a type shaft-bush joining by criterion of feeding and assembly of details without jamming into devices of automatic units are presented. Mathematical dependences can be used at a design stage of feeders, collectors and assembly systems of automatic devices and machines that allow enhancing their quality and efficiency of use.

\section{REFERENCES}

[1] Chow, We - Min, Assembly line design : methodology and applications, New York : Marcel Dekker , 1990.

[2] Beno Benhabib, Manufacturing. Design, Production, Automation, and Integration, Marcel Dekker Ink. 2003.

[3] Riley, Frank J. Ireson, Assembly automation : A management handbook 2nd ed. New York: Industrial Press , 1996.

[4] Elsayed, E.A., Reliability Engineers, AddisonWesley, 1996.

[5] G. Boothroyd, Handbook of Feeding and Orienting Techniques for Small Parts, Catalogue,
Department of Mechanical Engineering, University of Massachusetts, 1984.

[6] Groove, M.P, Automation Production Systems and Computer-Aided Manufacturing, Eaglewood Cliffs, NJ, Prentice Hall, 1980

[7] G. Boothroyd, Assembly automation and product design, Marcel Dekker Ink. 1992, $413 p$.

[8] Shafer, Dean A. Successful assembly automation : a development and implementation guide. Dearborn: Society of Manufacturing Engineers, 1998.

[9] Hajime Hitakawa, Advanced parts orientation system has wide application. Assembly Automation, 8(3), 1988.

[10] T. Suzuki and M. Kohno., The flexible parts feeder which helps a robot assembles automatically. Assembly Automation, 1981.

[11] Eastman CM. Design of assemblies; SAE technical paper series 0148-7191/81/0223-0197; Feb 23-27, 1981.

[12] R. Yoshida, M. Tajima, Versatile automatic feeding system with movable orienting attachment, in: Proceedings of 10th International Conference on Assembly Automation, Kanazawa, Japan, October 1989.

[13] Lee, K.-M., "Flexible Part-Feeding System For Machine Loading And Assembly. Part I. A State-OfThe-Art Survey," International Journal of Production Economics, 25, 1991, pp. 141-153.

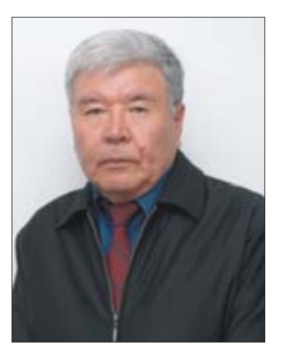

Prof. Ryspek Usubamatov is a Doctorate in Automation of Manufacturing Processes from Moscow State Technical University and a Professor at The School of Manufacturing Engineering, University Malaysia Perlis. He has received more then 60 patents of inventions in the area of automation of manufacturing processes, machine tools design, robotics, industrial automated transport systems, and technological processes. His fields of interests include Theory of Industrial and Manufacturing Productivity, Metal Cutting Manufacturing Processes, Industrial Machines Reliability, Automation of Manufacturing Processes and Inventions in different areas of human activity, CNC/CAD/CAM and Multi-axis Gear Machining on CNC Machining Centres. 